\title{
A VARIATIONAL FORMULATION FOR CONSTRAINED QUASILINEAR VECTOR SYSTEMS*
}

\author{
BY \\ NIMA GEFFEN \\ Tel-Aviv University
}

\begin{abstract}
A variational formulation for multi-dimensional initial- and/or boundaryvalue problems for a system of quasilinear conservation equations with a rotationality condition in a vector form with the aid of a vector Lagrange multiplier is given. The duality between the physical and 'phase' (or hodograph) spaces emerges, and the Lagrange multiplier turns out to be the vector potential for the conserved field, and hence of some interest in itself. Application is given to a family of transonic flows in the physical and hodograph planes, and to a problem in nonlinear sound propagation.
\end{abstract}

1. Introduction. Variational formulations equivalent to boundary-value problems for ordinary and elliptic partial differential equations have been of common use in particle and continuum mechanics and other branches of physics. For non-elliptic equations (e.g. diffusion and wave equations) the Dirichlet problem is improperly posed, and different conditions on parts of the boundary only have to be prescribed. Variational formulations for initial (rather than boundary) value problems have been proposed for linear parabolic and hyperbolic equations [1-5].

The two-dimensional nonlinear case was worked out in [6] with the aid of a Lagrange multiplier. It is extended here to three independent variables. The three-dimensional case is presented in vector notation which brings out the geometric and physical meaning of the various terms and the vector Lagrange multiplier, and is invariant under coordinate transformations. For more than three independent space variables, the constraints and Lagrange multipliers are skew-symmetric tensors.

The following analysis is applicable to fluid dynamics, plasma dynamics, nonlinear elasticity and nonlinear optics. Since the field does not have to be irrotational it can account for non-isentropic processes and shock waves. Applications are given to a number of transonic aerodynamic problems of much current interest (and some controversy) both in scientific research and technological developments, experimentation and design.

2. System of equations and variational formulation. Consider a solenoidal field $\mathbf{A}(\mathbf{u}$, $\mathbf{x})$ :

$$
\nabla \cdot \mathbf{A}=0
$$

where $\mathrm{u}$ is the vector of dependent variables and $x$ is the vector of independent variables, with a rotationality condition on $\mathbf{u}$ :

* Received September 29, 1976; revised version received January 28, 1977. Work supported in part by Grants AFOSR-73-2561 and AFOSR-76-2954; done in part at the University of Arizona. 


$$
\nabla \times \mathbf{u}=W
$$

and initial-boundary conditions:

$$
\left.u_{i}\right|_{\partial s_{i}}=g_{i}(x)
$$

prescribed on appropriate parts $\partial \Omega_{i}$ of the boundary $\partial \Omega$ of the region $\Omega$, where the solution $u$ is sought.

Eqs. (1), (2), (3) form a first-order quasilinear system of partial differential equations with initial and/or boundary conditions, assumed well posed, for the solution vector $\mathbf{u}(\mathbf{x})$. The mathematical structure and properties of solutions of the system (e.g. their degree of smoothness) depend on the type of the differential operator in (1), i.e. on the explicit functional form of $A(\mathbf{u}, \mathbf{x})$ which determines whether the system is elliptic, parabolic, hyperbolic or mixed. The way to prescribe the boundary conditions (3) for a given operator (1), (2) depends on the type of the operator, which will, in turn, determine what conditions $u_{i}=g_{i}$ on which part of its boundary $\Omega_{i}$ are to be prescribed (e.g. Dirichlet or Neuman data on the whole boundary $u_{i}=g_{i}, \sum_{i} \Omega_{i}=\Omega$ for an elliptic operator, Cauchy data $\mathbf{u}_{i}=\mathbf{f}_{i}$, on a non-characteristic plane $\Omega_{i} \subset \Omega$ and the remaining $\Omega_{m}=\Omega-\Omega_{i}$ without prescribed conditions for a hyperbolic one.)

The operator (1) is a conservation law for the field $A$, Eq. (2) is a rotationality condition on the solution vector $u(\mathbf{x})$ and Eq. (3) are 'outside' constraints to be satisfied whose form and extent are determined by the system modelled by Eqs. (1) and (2).

All functions are assumed smooth enough to make all expressions in Eqs. (1) and (2) defined uniquely in the weak sense. This requires that $\mathbf{u}(\mathbf{x})$ be continuously twice differentiable in all directions almost everywhere (i.e. $C^{2}$ a.e.) and that $A(\mathbf{u}, \mathbf{x})$ be once continuously differentiable in all its arguments $(\mathbf{u}$ and $\mathbf{x}$ ) almost everywhere (i.e., a.e.).

Theorem. a) The functional:

$$
J=\int_{\Omega}[L(\mathbf{v}, \mathbf{x})+\lambda \cdot(\nabla \times \mathbf{v}-\mathbf{W})] d \Omega+\int_{\partial \Omega m}(\lambda \times \mathbf{v}) \cdot d \mathbf{s}
$$

defined for functions $\mathbf{v}$ satisfying initial and boundary conditions (3) on $\partial \Omega_{i}$, and $\sum \partial \Omega_{m}$ $=\partial \Omega$, is stationary at the point

$$
\mathrm{u}=\mathbf{v},
$$

where $\boldsymbol{u}$ satisfies Eqs. (1) and (2) which form its Euler-Lagrange equations. Here

$$
\mathbf{A}=\nabla_{\mathbf{u}} L \text {, i.e. } \mathbf{A}_{i}=\partial L / \partial u_{i} .
$$

b) The Lagrangian $L$ exists if and only if

$$
\nabla_{(\mathbf{u})} \times \mathbf{A}=0
$$

c) The vector Lagrange multiplier $\lambda_{(}(\mathbf{x})$ is related to $\mathbf{A}$ by:

$$
\mathbf{A}=-\nabla \times \lambda
$$

and $\lambda$ is held fixed or its increment $\delta \boldsymbol{\partial}$ is kept proportional to $\mathbf{v}$ on the free boundary $\partial \Omega_{m}$.

Proof. Calculating the variation of $J$, one gets:

$\delta J=\int_{\Omega l}[\delta L+\lambda \cdot(\nabla+\delta \mathbf{v})+\delta \lambda \cdot(\nabla \times \mathbf{v}-\mathbf{W})] d \Omega+\int_{\partial \Omega m}(\lambda \times \delta \mathbf{v}) \cdot d \mathbf{s}+\int_{\partial \Omega m}(\delta \lambda \times \mathbf{v}) \cdot d \mathbf{s}$ 
$=\int_{\Omega}\left[\nabla_{v} L \cdot \delta \mathbf{v}+\lambda \cdot(\nabla \times \delta \mathbf{v})+\delta \lambda \cdot(\nabla \times \mathbf{v}-\mathbf{W})\right] d \Omega+\int_{\partial \Omega \Omega_{m}}(\lambda \times \delta \mathbf{v}) \cdot d \mathbf{s}+\int_{\partial \Omega \Omega_{m}}(\delta \lambda \times \mathbf{v}) \cdot d \mathbf{s}$

Using the vector relation

$$
V \cdot(\lambda \times \delta \mathbf{v})=-\lambda \cdot(\nabla \times \delta \mathbf{v})+\delta \mathbf{v} \cdot(\nabla \times \lambda)
$$

and Green's theorem

$$
\int_{\Omega} V \cdot(\lambda \times \delta \mathbf{v}) d \Omega=\int_{\partial \Omega}(\lambda \times \delta \mathbf{v}) \cdot d \mathbf{s}
$$

one gets:

$$
\begin{aligned}
\delta J= & \int_{\Omega \Omega}\left[\left(\nabla_{v} L+\nabla \times \lambda\right) \cdot \delta \mathbf{v}+(\nabla \times \mathbf{v}-\mathbf{W}) \cdot \delta \lambda\right] d \Omega-\int_{\partial \Omega \Omega}(\lambda \times \delta \mathbf{v}) \cdot d \mathbf{s} \\
& +\int_{\partial \Omega ! m}(\lambda \times \delta \mathbf{v}) \cdot d \mathbf{s}+\int_{\partial \Omega m}(\delta \lambda \times \mathbf{v}) \cdot d \mathbf{s}=0 .
\end{aligned}
$$

Since all v's satisfy given conditions on $\partial \Omega_{i}:\left.\delta \mathbf{v}\right|_{\partial \infty m}=0$ and since $\partial \Omega_{m}=\partial \Omega-\partial \Omega_{i}$, the first two surface integrals cancel; the last surface integral vanishes when either $\lambda$ is fixed on $\partial \Omega_{m}$ or when $\delta \lambda$ is parallel to $\mathbf{v}$. Hence, writing $\mathbf{u}$ for $\mathbf{v}$ by the fundamental lemma of the calculus of variations, we obtain

$$
V_{\mathrm{u}} L+\nabla \times \lambda=0
$$

and

$$
\nabla \cdot\left(\nabla_{\mathbf{u}} L\right)=0
$$

or:

$$
\mathbf{A}=\nabla_{\mathbf{u}} L
$$

In addition,

$$
\cdot \nabla \times \mathbf{u}-W=0 ;
$$

i.e. Eqs. (1) and (2) are recovered as the Euler-Lagrange equations of the functional. Since $\mathbf{A}$ is a gradient in $\mathbf{u}$ space it is irrotational there, i.e.:

$$
\nabla_{(\mathbf{u})} \times \mathbf{A}=0 .
$$

and the vector Lagrange multiplier $\lambda$ is the vector potential of the field $\mathbf{A}$ :

$$
\mathbf{A}=-\nabla \times \lambda \text {. }
$$

Eq. (2) for $\lambda$ inside the domain $\Omega$ and the boundary condition required for the vanishing of the surface integral on the "free" boundary are compatible. The surface term is a "penalty" term which adjusts the assumed Lagrange multiplier $\lambda(\mathbf{v}, \mathbf{x})$ for a trial field $\mathbf{v}$ to the vector potential of $\mathbf{A}$ for the solution field $\mathbf{u}(\mathbf{x})$ at the free boundary where $\mathbf{u}$ (hence v) is not prescribed.

The domain $\Omega$ with its boundary $\partial \Omega=\partial \Omega_{i}+\partial \Omega_{m}$ is assumed regular and smooth enough for Green and Gauss volume-to-surface integrals to hold. The trial functions $v$ are smooth enough to make all terms in the integrand defined almost everywhere (which, in general, will require $\mathbf{v} \in C^{1}(\mathbf{x})$ a.e. and the integral $J(\mathbf{v})$ definite and finite). 
3. Remarks on some features of the system. The solenoidal field A (Eq. (1)) has to be irrotational in u-space (Eq. (6)) for existence of a variational formulation of the postulated type. Mathematically, this insures the formal symmetry of the Gateaux derivative [7] of the operator (1), which, in turn, is the condition for the existence of a Lagrangian, and stationarity of the functional $J$. The Lagrangian $L$ is the potential in $\mathrm{u}$ space of the vector field $\mathbf{A}(\mathbf{u}, \mathbf{x})$.

The vector Lagrange multiplier $\lambda$ (Eq. (7)) in the present analysis turns out as the vector potential of the solenoidal field $\mathbf{A}$, or the stream function for the two-dimensional case [6], and hence is of interest in itself.

Finally, in view of Eqs. (5) and (7) relating the vector field $\mathbf{A}$ to a scalar and vector potentials in $\mathbf{u}$-space, the variational formulation is not unique; an arbitrary $f(x)$ and $x$-gradient can be added to $L$ and $\lambda$ respectively, i.e.:

$$
\begin{gathered}
L^{\prime}=L+f(\mathbf{x}), \\
\lambda^{\prime}=\lambda+\nabla g(\mathbf{u}, \mathbf{x}),
\end{gathered}
$$

thus giving a set of equivalent variational principles for a given problem (gauge invariance) presumably with a unique solution.

The variational formulation above is invariant under coordinate transformations. One of the $\mathbf{x}$-components can be time, and the boundary term in the expression for $J$ (Eq. (4)) can account for appropriate initial conditions for time-dependent problems (e.g. the nonlinear wave equation [6]). Since the vector-field $u$ need not be irrotational (Eq. (2)), nonisentropic flow discontinuities of the shock-wave type are admissible in the solutions. The variational formulation provides a convenient basis for approximations, e.g. by the Rayleigh-Ritz method or in finite-element spaces.

4. Application to steady and unsteady transonics. The equation

$$
\left(a+b y+c \phi_{x}\right) \phi_{x x}+\phi_{y y}+\alpha \phi_{z z}=0,
$$

where $(a, b, c, \alpha)$ are given constants, describes a variety of small-perturbation transonic flows. It is nonlinear, of a mixed hyperbolic-elliptic type, and the appropriate initial and/or boundary conditions depend on its type, which, in turn, depends on the signs of the coefficients of its highest derivatives.

Writing (11) as a first-order system one gets:

$$
\begin{gathered}
\mathbf{u}=(u, v, w)=\left(\phi_{x}, \phi_{y}, \phi_{z}\right) ; \quad \nabla \times \mathbf{u}=0 \\
(a+b y+c u) u_{x}+v_{y}+\alpha w_{z}=0
\end{gathered}
$$

or in a conservation form:

$$
\left(a u+b y u+c u^{2} / 2\right)_{x}+v_{y}+\alpha w_{z}=0 .
$$

Hence

$$
\mathbf{A}=\left[A_{1}, A_{2}, A_{3}\right]=\left[a u+b y u+\frac{c}{2} u^{2}, v, \alpha w\right]
$$

and since each $A_{i}$ depends only on the corresponding component $u_{i}$,

$$
\nabla_{\mathbf{u}} \times \mathbf{A}=0
$$

and the Langrangian of the system is: 


$$
L=a \cdot \frac{u^{2}}{2}+b y \frac{u^{2}}{2}+\frac{c}{2} \frac{u^{3}}{3}+\frac{1}{2}\left(v^{2}+\alpha w^{2}\right)
$$

which can be either positive, negative, or indefinite; e.g., $L>0$ for $u>0, \alpha>0$ for which (11) is elliptic, while for $u \lessgtr 0, L$ is indefinite. The variational formulation of the system is:

$$
\begin{aligned}
J(\mathbf{u}, \lambda) & =\iiint_{\Omega(x, y, z)}\left[a \frac{u^{2}}{2}+b y \frac{u^{2}}{2}+\frac{c}{2} \frac{u^{3}}{3}+\frac{1}{2}\left(v^{2}+\alpha w^{2}\right)+\lambda \cdot \nabla \times \mathbf{u}\right] d x d y d z \\
& +\iint_{\partial \Omega l_{m}} \lambda \times \mathbf{u} \cdot \mathbf{d s}
\end{aligned}
$$

where

$$
\begin{aligned}
\lambda & =\left(\lambda_{1}, \lambda_{2}, \lambda_{3}\right) ; \quad \mathbf{u}=(u, v, w), \\
\nabla \times \mathbf{u} & =\left[\left(w_{y}-v_{z}\right),\left(u_{z}-w_{x}\right),\left(v_{x}-u_{y}\right)\right], \\
\lambda \cdot \nabla \times \mathbf{u} & =\lambda_{1}\left(w_{y}-v_{z}\right)+\lambda_{2}\left(u_{z}-w_{x}\right)+\lambda_{3}\left(v_{x}-u_{y}\right), \\
\lambda \times \mathbf{u} & =\left[\left(\lambda_{2} w-\lambda_{3} v\right),\left(\lambda_{3} u-\lambda_{1} w\right),\left(\lambda_{1} v-\lambda_{2} u\right)\right], \delta J(\mathfrak{u}, \lambda)=0 .
\end{aligned}
$$

Case a. Nonlinear acoustic behavior at a caustic [8]. Setting $a=c=0, b=\alpha=1$ we get a three-dimensional version of Tricomi's equation, widely investigated in the context of the hodograph representation of two-dimensional transonic flows. With $c=\epsilon$ a small nonlinear perturbation thereof is obtained. Its two-dimensional version (i.e., $\alpha=0$ ) has been extensively investigated, both analytically and numerically, by Seebass (e.g., [8]). The equation:

$$
-\left(y+\epsilon \phi_{x}\right) \phi_{x x}+\phi_{y y}+\phi_{z z}=0
$$

is hyperbolic for $y+\epsilon \phi_{x}>0$ and elliptic for $y+\epsilon \phi_{x}<0$. In analogy to the linear twodimensional case, we seek a solution in a domain $(x, y, z)$ bounded by $\partial \Omega=S_{1}$ in the elliptic part and $S_{2}$ in the hyperbolic one. Consider a mixed domain intersecting the $y=0$ axis along the rectangle $A A^{\prime} B B^{\prime}$, where $x_{A} \leq x \leq x_{B}, 0 \leq z \leq 1$. For this case $S_{1}$ is an arbitrary, sufficiently smooth, surface in the lower $(y<0)$ half space and $S_{2}$ is bounded by the characteristic "roof" $A C B B^{\prime} C^{\prime} A^{\prime} A$ (Fig. 1) and the "vertical" parts $(z=0) A C B,(z=$ 1) $A^{\prime} C^{\prime} B^{\prime}$.

$A C: \quad x-\frac{2}{3} y^{3 / 2}=0$

$B C: x+\frac{2}{3} y^{3 / 2}=0$

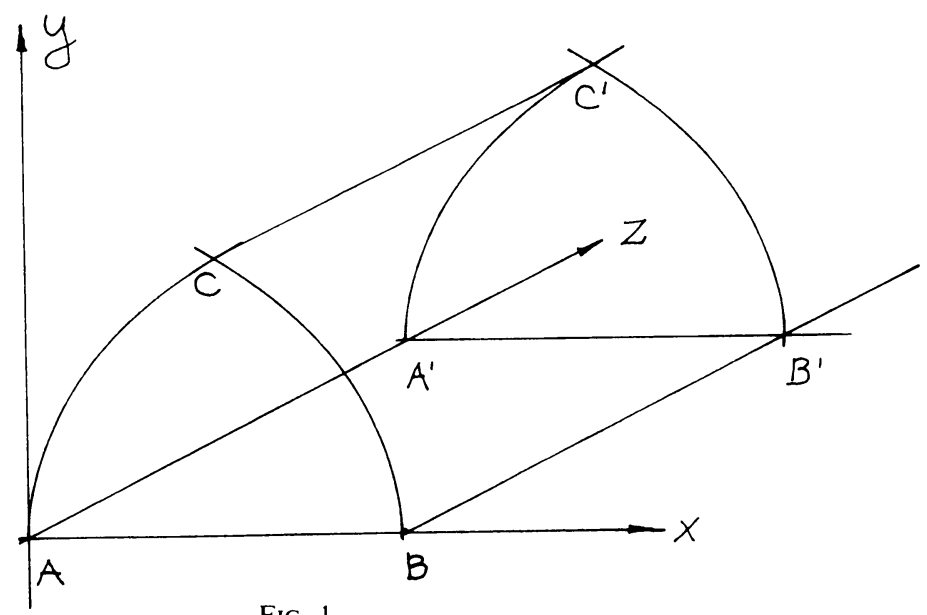

FIG. 1. 
The first-order system is:

$$
-(y+\epsilon u) u_{x}+v_{y}+w_{z}=0 ; \quad \nabla \times \mathbf{u}=0
$$

with no boundary conditions prescribed on the characteristic surface $B C C^{\prime} B^{\prime} \equiv \partial \Omega_{m}$; hence we obtain the variational formulation

$J(u, \lambda)=\iiint_{\Omega(x, y, z)}\left[-\left(y u+\frac{\epsilon}{2} \frac{u^{3}}{3}\right)+\frac{1}{2}\left(v^{2}+w^{2}\right)+\lambda \cdot \nabla \times \mathbf{u}\right] d x d y d z+\int_{\partial \Omega_{m}} \lambda \times \mathbf{u} \cdot \mathbf{d s}$

where ds $=d z(d y,-d x(y), 0)$ with $(x, y)$ along $B C$ and

$$
\begin{aligned}
\int_{\partial \Omega l_{m}} \lambda \times \mathbf{u} \cdot \mathrm{ds} & =\int_{0}^{z_{B^{\prime}}} \int_{0}^{y_{c}}\left[-\left(\lambda_{2} w-\lambda_{3} v\right) d y+\left(\lambda_{3} u-\lambda_{1} w\right) d x(y)\right] d z \\
& =\int_{0}^{z_{B^{\prime}}} \int_{0}^{y_{c}}\left[\lambda_{3}(u d x+v d y)-w\left(\lambda_{1} d x+\lambda_{2} d y\right)\right] d z
\end{aligned}
$$

Case b. Three-dimensional flow about a body [9]. Setting $b=0, \alpha=1$, we get the standard small-perturbation three-dimensional transonic equation of, say, uniform flow around a slender body.

$$
\left(a+c \phi_{x}\right) \phi_{x x}+\phi_{y y}+\phi_{z z}=0 .
$$

Let the region considered be

$$
(|x|,|y|,|z|) \leq(|\bar{x}|,|\bar{y}|,|\bar{z}|)
$$

where $\bar{x}, \bar{y}, \bar{z}$ may be infinite, with a "hole" (or slit), $Y=Y(x, z) ;(x, z) \in A: y=0$, describing the body. Boundary conditions are given for the far-field $(\bar{x}, \bar{y}, \bar{z})$ (e.g. vanishing potential and its derivatives, or appropriate asymptotic solution) and partial boundary conditions on the body, e.g.:

$$
\nabla[X-Y(x, z)] \cdot(1+\nabla \phi)=0
$$

or

$$
a \phi_{x}+b \phi_{y}+c \phi_{z}+d=0 .
$$

The first-order system for this case is

$$
(a+c u) u_{x}+v_{y}+w_{z}=0 ; \quad \nabla \times \mathbf{u}=0,
$$

and the variational formulation is

$$
J=\int_{-\bar{z}}^{\bar{z}} \int_{-\bar{y}}^{\bar{y}} \int_{-\bar{x}}^{\bar{x}}\left[\mathrm{au}+\frac{c}{2} \frac{u^{3}}{3}+\frac{1}{2}\left(v^{2}+w^{2}\right)+\lambda \cdot \nabla \times \boldsymbol{u}\right] d x d y d z+\int_{\partial \Omega_{m}}
$$

where

$$
\begin{gathered}
\partial \Omega_{m}=Y(x, y=0), \mathrm{ds}=(0, d x d z, 0), \\
\int_{\partial \Omega_{m}}=\left.\int_{x, z} \int_{\in A}\left(\lambda_{3} u-\lambda_{1} w\right)\right|_{y=0} d x d z .
\end{gathered}
$$




\section{REFERENCES}

[1] M. E. Gurtin, Variational principles for linear initial-value problems, Quart. Appl. Math. 22, 252-256 (1964)

[2] B. Noble, Variational finite element methods for initial value problems, in The mathematics of finite elements applications, J. R. Whiteman, ed., Academic Press, 1973

[3] E. Tonti, On the variational formulation for linear initial-value problems, Ann. Math. Pura Appl. 95, 33̣1-360 (1973)

[4] I. Herrera and J. Bielak, A simplified version of Gurtin's variational principles, J. Rat. Mech. 53, 131-149 (1974)

[5] J. N. Reddy, A note on mixed variational principles for initial value problems, Quart. J. Mech. Appl. Math. 28, 123-132 (1975)

[6] N. Geffen and S. Yaniv, A note on the variational formulation for quasilinear initial value problems, ZAMP.

[7] I. Herrera and J. Bielak, Dual variational principles for diffusion equations, Quart. Appl. Math 34, 85-102 (1976)

[8] N. J. Yu and R. Seebass, Computational procedures for mixed equations with shock waves, in Computational methods in nonlinear mechanics, T. J. Oden, ed., 1974, pp. 499-508.

[9] H. Lomax, R. Bailey, and W. F. Ballhaus, On the numerical simulation of three-dimensional transonic flow with application to the C-14I wing. NASA TN D-6933, August 1973 